\title{
La formación de investigadores-profesores en la calidad de la educación superior en México.
}

\author{
The training of researchers-professors in the quality of higher education in \\ Mexico
}

\author{
Angélica Mendieta Ramírez \\ Benemérita Universidad Autónoma de Puebla \\ angelicamendietaramirez@yahoo.com.mx \\ Alain Pérez Martínez \\ Benemérita Universidad Autónoma de Puebla \\ alainpm@hotmail.com
}

\section{Resumen}

Resulta importante abordar el impacto de la formación de investigadores-profesores en la calidad de la educación superior en México, debido a que actualmente parece estar en crisis -cabría preguntarse si ¿en algún momento no ha estado en crisis la formación de investigadores-profesores? Es probable que el estado natural de la calidad educativa sea el de un constante desequilibrio debido a que la calidad en educación debe responder al desarrollo integral formativo, a la sociedad y al mercado laboral, entre otros; y al hecho de estar inmersos en una realidad tan cambiante que nos enfrentamos a constantes retos, necesidades y exigencias, es decir, económicas, políticas y sociales; así como en sus diversas versiones tanto internacionales, nacionales, estatales, locales. Todo ello ha traído como consecuencia una desalineación, la cual pudiera explicarse por la pérdida de los objetivos centrales propios de la Universidad y de su modelo educativo. 
Palabras clave: Formación, Investigadores, Calidad Educativa, Universidad, Docencia, Competitividad Educativa, Modelo Educativo.

\section{Abstract}

It is important to address the impact of the training of research-quality teachers in higher education in Mexico, because at present seems to be in crisis, one might wonder whether did you ever not been in crisis training researchers-teachers? It is likely that the natural state of educational quality is that of a constant imbalance because the quality of education must respond to the comprehensive development training, society and the labor market, among others, and the fact of being immersed in a reality changing as we face constant challenges, needs and requirements, ie, economic, political and social, as well as in its various versions both international, national, state, local. All this has led to a misalignment, which could be explained by the loss of the central objectives specific to the University and its educational model.

Key words: Training, Researchers, Quality Education, University, Education, Educational Competitiveness, Educational Model.

Fecha recepción: Julio 2012

Fecha aceptación: Diciembre 2012

\section{Introducción}

Uno de los problemas que actualmente impera en las universidades públicas y privadas es la falta de correspondencia entre los programas educativos y las demandas laborales y sociales, esto puede deberse a que el profesorado no está actualizado en la teoría y práctica de su formación, trayendo como consecuencia graves problemas como la escasa lectura e investigación que lo hagan involucrarse en las diversas corrientes del pensamiento teórico-epistemológico que lo conduzca a propuestas de mejora y la 
resolución de problemas reales (casos concretos) o bien que contribuyan a la generación del conocimiento.

Es importante señalar que también el docente se encuentra obstaculizado en su práctica educativa por los factores endógenos y exógenos, encontrándose la mayoría de las veces con grupos muy numerosos, la falta de una preparación pedagógica que le permita orientar la formación integral de los estudiantes y si a esto le sumamos el excesivo número de horas-pizarrón. De aquí que nos hacemos las siguientes preguntas ¿hacia dónde debe orientarse la formación de investigadores-profesores de educación superior? y ¿qué papel juega la investigación para la formación en la docencia?

Estas interrogantes nos conducen a plantear la siguiente hipótesis Para formar un investigador docente en una universidad pública o privada es menester que el profesor sea reflexivo, analítico, crítico y propositivo de su entorno; para ello es indispensable trasladarnos de un pensamiento clásico y reduccionista de la realidad a un pensamiento complejo, abierto, holográmatico, creativo y humanista. Esto requiere empezar a desaprender para aprender.

\section{Formación de investigadores-profesores}

Un estudio realizado por el Consejo para la Acreditación de la Educación Superior, (COPAES: 2003) señala que el sistema de educación superior presenta varios problemas que afectan la calidad de ese nivel educativo en México. El Programa Nacional de Educación 2001-2006, elaborado por la Secretaría de Educación Pública, expone un diagnóstico de la problemática de cada nivel educativo y es, al mismo tiempo, la guía que orienta el trabajo para superar los obstáculos. En lo que se refiere a la educación superior 
éstos son los problemas señalados: Rigidez en los programas educativos: Debilidades de los cuadros académicos, sólo una parte del profesorado del nivel superior labora tiempo completo, y de ellos, el $11 \%$ produce conocimiento, cuyo indicador es la publicación de artículos de investigación en revistas de circulación internacional (SEP: 2006), insuficiente producción de conocimiento aun cuando la producción científica del país se ha triplicado en el último decenio, la contribución al total mundial al año no llega al 1\%, lo cual es inferior a lo esperable para una economía con las dimensiones de la mexicana. "En las instituciones públicas de educación superior se realiza la mayor parte de la investigación científica y humanística del país. Sin embargo, la capacidad institucional para la investigación está distribuida muy heterogéneamente en el territorio nacional y su debilidad en muchas de las dependencias e instituciones que por su misión deberían cultivarla afecta la calidad de los programas educativos".

La universidad del siglo XXI debería ofrecer programas de formación profesional con currículos caracterizados por: flexibilidad en su diseño y ejecución; rigor académico en el trabajo curricular, desde el diseño hasta la evaluación integral del currículo; enfoques multidisciplinarios y ejecución efectiva; integración cultural y multicultural en su orientación.

Algunas universidades inglesas valoran la idoneidad del profesorado universitario (los profesores deben acreditarse para poder adquirir la condición de profesorado fijo en algunas universidades) en base a 5 competencias: organización, presentación, relaciones, tutoría-apoyos a los estudiantes, evaluación. Y, si desean adquirir la condición de profesores excelentes, se eleva el número de competencias y el nivel de exigencia. Los candidatos a la excelencia docente deben acreditar la posesión de las siguientes competencias: las 5 ya mencionadas pero en un nivel superior de dominio; reflexión, innovación, capacidad de desarrollo curricular; organización de cursos, investigación pedagógica y, finalmente, liderazgo de grupo. 
En este sentido, es importante precisar que el trabajo de Perrenoud (2004) y sus 10 competencias del profesor (no se refieren al profesor universitario). Otra propuesta es la de Cano (2005). Lo que no se debe perder de vista es el sentido de la docencia como vocación y compromiso personal, puesto que son fundamentales en el proceso formador del docente, porque enseñar es una tarea compleja que requiere de conocimientos, de recursos tecnológicos para enriquecerla y de una clara perspectiva ética de su quehacer docente. En este sentido se habla hoy de la formación de formadores.

\section{La Calidad educativa}

En este trabajo entendemos que la calidad de un sistema de educación, en este caso superior, es resultante de un proceso histórico en el que se han conjugado factores tanto endógenos como exógenos a las instituciones, que han afectado el desarrollo de las funciones académicas. La calidad no hace sólo a la mejora en la eficiencia o eficacia, en el logro de los productos deseados, sino también a la definición cualitativa de esos mismos deseos, expresados en objetivos o finalidades de la educación, en congruencia con las necesidades o proyectos nacionales (ANUIES: 1987).

El Consejo para la Acreditación de Programas Educativos en Humanidades A.C (2007), entiende por calidad de la educación superior la combinación congruente, tanto de la aspiración de potenciar valores y posibilidades del ser humano (autonomía, crítica, libertad, creatividad, autoaprendizaje, posibilidad de elección, equidad, sentido de la justicia, altruismo, sensibilidad estética, posibilidad de dar sentido al pensar y al hacer humano, capacidad de reconocimiento, respeto y fomento de la alteridad, así como apertura de diálogo por medio de razones), como del aseguramiento de los procesos académicos que garanticen niveles adecuados de eficiencia terminal, de retención de estudiantes, desarrollo de investigaciones originales, intercambio académico, actualización curricular, interacción social y difusión cultural. Este organismo de acreditación pretende desarrollar estrategias que le permitan constatar el modo en que las IES del área implementan en su desempeño este concepto de calidad o aspectos 
importantes del mismo. La acreditación, en su connotación institucional e individual, implica una búsqueda de reconocimiento social y de prestigio tanto por parte de los individuos que transitan por las instituciones educativas, como por estas mismas. En este sentido los procesos de acreditación se han constituido en un requerimiento necesario en nuestros días, en virtud de que garantizan la calidad y credibilidad de un proceso educativo y de sus resultados (Pallán, 1995). De ahí que la acreditación tenga un papel estratégico dentro de la política educativa orientada a promover cambios significativos en la organización y eficiencia del sistema de educación superior (Pallán, 1995).

Por lo anterior, esta investigación no busca definir la calidad educativa a nivel superior, sino más bien contextualizarla y encontrar los elementos esenciales que la identifican, evalúan y valoran. Entendemos con Diestro Fernández, que no obstante que se haya discutido el término en repetidas ocasiones, preexiste una indefinición de éste. Situación que se hace comprensible debido a la complejidad de la misma, suele estar vinculado en una multitud de fines, que ahora no analizaremos, los cuales no siempre están encaminados a la mejora del proceso educativo. Por lo que se plantean o agrupan las definiciones de la calidad educativa en cuatro grandes tendencias:

1. Las que se centran en los resultados (el producto) Identifican la calidad educativa con un producto funcional, válido, competitivo y eficiente.

2. Las que se centran en el proceso. La definición de Esteban y Montiel lo aclara: "Proceso o principio de actuación que no apunta exclusivamente a la obtención de unos resultados inmediatos o finales, sino, fundamentalmente, a un modo de ir haciendo, poco a poco, las cosas para alcanzar los mejores resultados posibles en orden a lo que se nos demanda y a las posibilidades y limitaciones reales que se tiene"

3. Los posicionamientos eclécticos (la coherencia) La calidad entendida como forma inherente al proceso y al producto. (Integridad, coherencia y eficacia) 
4. La visión empresarial de la educación. Proporcionar a los clientes (padres, hijos, tutores, AMPAS, etc.) los productos y servicios que satisfagan plenamente los requerimientos acordados.

De todo ello deducimos que la concepción del término calidad de la educación está estrechamente relacionada con la idea de educación que se tenga. Una persona de tendencia liberal, moderada, progresista o marxista tendrá una concepción diferente del término calidad. Por tanto, la definición dependerá del sujeto que evalúa, de los objetos o procesos comparados, de la ideología a la que responda, de las expectativas, de las exigencias sociales, de la visión económica en la que se concibe, de la institución o empresa que la pretenda, etc., también resulta importante precisar que el paradigma cuantitativo y cualitativo en este proceso ha sido y sigue siendo el centro de la discusión, pero la definición tendrá que situarse al amparo de alguna de las tendencias anteriormente citadas (Diestro Fernández, 2003).

Sin embargo, estas tendencias han tenido que ajustarse a las reformas o modernizaciones de los sistemas educativos regionales y nacionales de los Ministerios, Secretarías de Estado y los nuevos Modelos Educativos de las Universidades se han replanteado en esta lógica siguiendo las directrices de la UNESCO, BM, FMI, OCDE, con su Documento DeSeCo (Definición y selección de competencias'), etc., específicamente, con el proyecto EEES (Espacio Europeo de Educación superior) y sus 'Plan Bolonia, la Declaración de Glasgow (de las Universidades Europeas), Bergen, Londres, el CERI (Centro para la investigación e innovación educativas), los Proyectos para Europa y A. Latina: con sus proyectos SócratesErasmus-Leonardo da Vinci, Tuning, 6x4 (UE-AL Caribe) , Alfa-Europa, (ya Alfa III E/AL con su Innova-Cesal),

Detrás de todos estos proyectos de modernización educativa que exigen cambios no solamente en los Modelos Educativos de las Universidades en México, es decir, se requiere algo más que buenas intenciones fundamentadas en las teorías pedagógicas de la 
educación, sino más bien se hace evidente el divorcio de ideas y prácticas educativas propias de la escuela tradicional. Esto ha ido integrando la llamada 'industria del aprendizaje por competencias'.

Dado lo anterior y tomado en cuenta la necesidad de identificar los elementos que permitan ponderar de alguna manera la calidad de la educación superior en México, se ha considerado importante consultar los resultados del Índice de Transparencia y Acceso a la Información de las Universidades Públicas (ITAIUP: 2008), cuya medición abarca a 39 Universidades Públicas en el país, de lo cual se puede destacar que los servicios educativos en donde México ha mostrado importantes debilidades, como lo muestran diversas investigaciones que señalan la baja cobertura educativa a nivel superior, pues de cada 10 mil habitantes, sólo tienen acceso a educación pública superior 225, ubicándose en el lugar 15 en América Latina, por debajo de Argentina, Panamá, Costa, Rica, Venezuela, Chile, Bolivia, República Dominicana, Perú, Granada, Uruguay, Barbados, Antigua y Bermuda, Cuba y Colombia, ello a pesar de ser, la nación que más gasto público ejerce por estudiante en América Latina.

Es importante destacar que si comparamos el gasto por alumno en educación superior entre México y algunos países de la región, observamos que existe una diferencia importante en el monto asignado, ya que mientras que México destinó 4,289 dólares por alumno, Chile y Argentina destinaron poco más de 3,500 dólares por alumno en el mismo año (OCDE: 2006) y seis años después aumento 47 por ciento, es decir, el gasto anual por estudiante fue de 8,020 dólares en México (OCDE: 2012). Esto nos hace pensar en la falta de capacidad administrativa y de distribución de los recursos económicos para generar espacios educativos adecuados tanto en infraestructura como en la calidad educativa mexicana. México destina la segunda proporción más alta de este gasto a la educación, el 20.3 por ciento, después de Nueva Zelanda, mientras que los países de la organización invierten el 13 por ciento, pero México logra igualar el gasto promedio en proporción al PIB de los países de la organización. El gasto educativo en México es de 6.3 por ciento del 
Producto Interno Bruto, lo que coloca al país por encima del promedio de lo que presupuesta el resto de las naciones en el sector.

En los últimos nueve años el número de universidades privadas creció a tasas de entre 4.5 y 5\%, pasando a cerca de mil 600 instituciones, en las que se imparten más de 21 mil 100 licenciaturas o posgrados, pero en casi la mitad de ellas no existe una evaluación que avale la calidad del servicio que ahí se brinda. La Secretaría de Educación Pública (SEP: 2009) establece que de esas universidades, sólo 538 tienen reconocimiento por su buena calidad.

Los registros que tiene la SEP, de 1991 a 2009, señalan que mientras el número de jóvenes que se incorporaron a las universidades públicas se duplicó; en el caso de las universidades privadas, el número de estudiantes se cuadruplicó en el mismo periodo. Al pasar de poco menos de 250 mil a casi un millón de alumnos. La SEP a su vez señala que hay rezago histórico porque sólo podrán asistir a la universidad a 28 de cada cien jóvenes en México.

Se puede considerar que la Universidad es escasamente eficaz y funcional si solamente logra algunos de los objetivos con alta significación social y falla en otros a causa de una deficiente distribución y uso de recursos docentes y de investigación. La calidad, en esta perspectiva, aparece como un continuo de forma escalar, cuyos puntos representan combinaciones de funcionalidad, eficacia y eficiencia, mutuamente implicados. Su grado máximo, la excelencia, supone un óptimo nivel de coherencia entre todos los componentes principales representados en el modelo sistémico. 
(Dado el enfoque sistémico adoptado, la calidad de la educación superior supone una

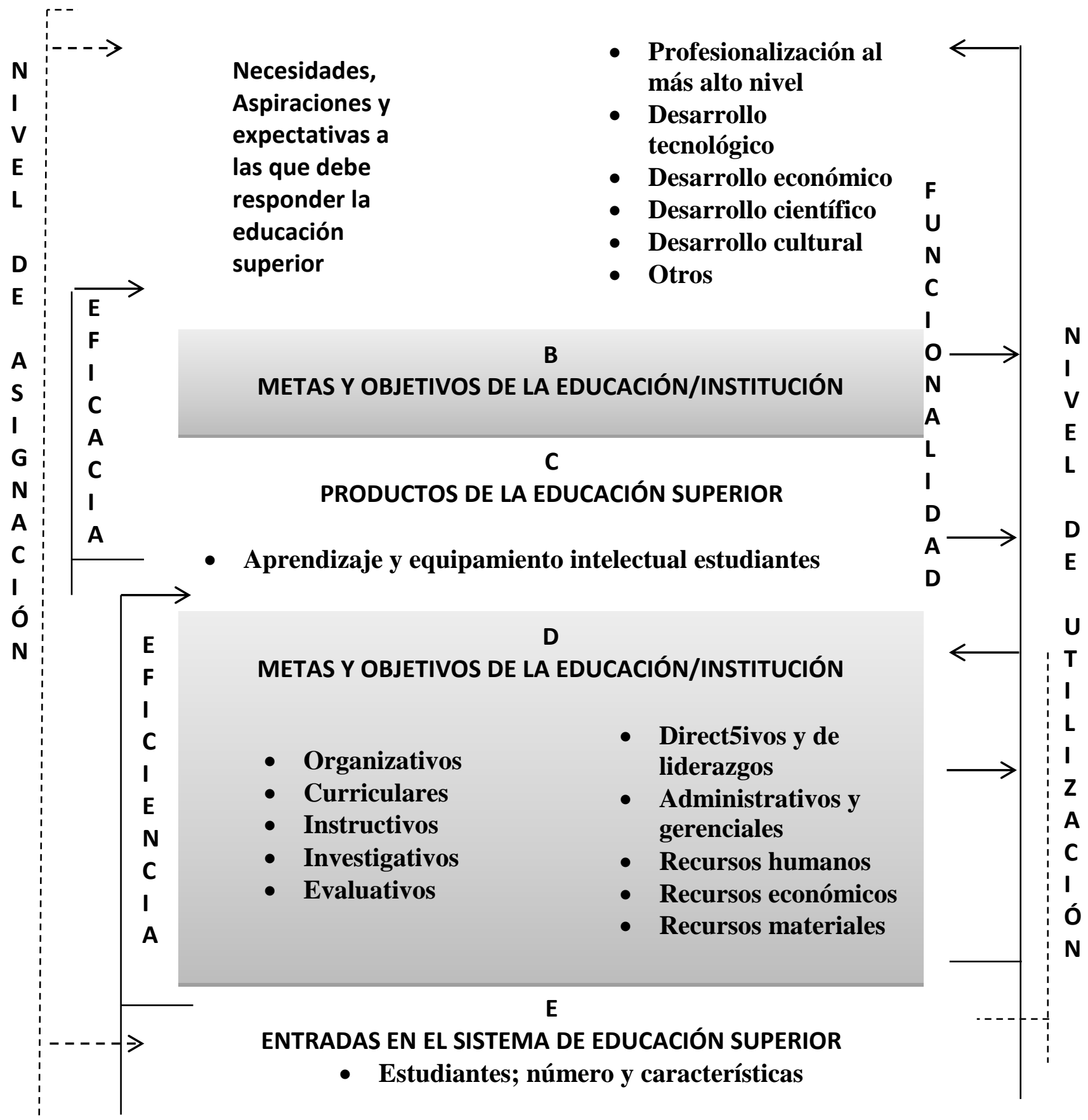

(De la orden, 1997)

relación de coherencia entre cada uno de los componentes del

sistema. En unos casos, esta relación de coherencia o incoherencia será evidente, dada la 
proximidad estructural y/o funcional entre los componentes relacionados. Tal es el caso, por ejemplo, de la relación postulada entre "Metas y objetivos de la educación universitaria" (B) y las "Necesidades sociales" (A); o entre "Productos de la educación universitaria" (C) y "Metas y Objetivos" (B). En estos casos, la relación aparece como directa e inmediata. En otros casos, la relación sería menos evidente, como, por ejemplo, la supuesta entre "Procesos de gestión" (D) y "Necesidades sociales" (A). Aquí se trata de relaciones indirectas y mediatas. Pero cualquier ruptura en la red de coherencias entre componentes supondría una limitación más o menos severa de la calidad educativa. (De la Orden, 1997). Según esta teoría, el objetivo del sistema es la formación de profesionales que respondan a las necesidades y expectativas económicas y sociales, en tanto que la calidad de la educación universitaria se identifica con un complejo constructo explicativo de valoraciones, apoyado en la consideración conjunta de tres dimensiones interrelacionadas: funcionalidad, eficacia y eficiencia, expresión, a su vez, de un conjunto integrado de relaciones de coherencia entre los componentes básicos de la educación o de una institución universitaria concebidos como un sistema. En primer lugar, la coherencia entre, por un lado, inputs, procesos, productos y metas y, por otro, expectativas y necesidades sociales define la calidad de la educación universitaria como funcionalidad. En segundo lugar, la coherencia del producto con las metas y objetivos define la calidad de la educación universitaria como eficacia o efectividad. En tercer lugar, la coherencia entre, por un lado, input y procesos y, por otro, producto, define la calidad de la educación universitaria como eficiencia. Dentro del modelo, carece de sentido hablar de eficiencia, en ausencia de eficacia, y es dudoso considerar como eficaz una institución universitaria que logra unos objetivos poco relevantes para los estudiantes y para la sociedad, es decir, con un bajo nivel de funcionalidad. Por otra parte, una universidad será considerada escasamente eficaz y funcional si solamente logra algunos de los objetivos con alta significación social y falla en otros a causa de una deficiente distribución y uso de recursos docentes y de investigación. La calidad, en esta perspectiva, aparece como un continuo de forma escalar, cuyos puntos representan combinaciones de funcionalidad, eficacia y eficiencia, mutuamente implicados. Su grado máximo, la 
excelencia, supone un óptimo nivel de coherencia entre todos los componentes principales representados en el modelo sistémico. (De la Orden, 1997).

-

Modelo Heurístico de enseñanza - aprendizaje de Entwistle (1987):

Este modelo pone énfasis en la relación de tres componentes al interior de la actividad docente:

2.

Enfoque Sistémico: La Teoría de Sistemas plantea que las organizaciones son sistemas abiertos, que a su vez son subsistemas de la sociedad en que están insertos. Las organizaciones y la sociedad se relacionan por medio de los objetivos que constituyen su función social.

En este contexto que surge la acreditación como un proceso por medio del cual un programa o institución educativa brinda información sobre sus operaciones y logros a un organismo externo que evalúa y juzga, de manera independiente, dicha información para poder hacer una declaración pública sobre el valor o la calidad del programa o de la institución.

Por su parte la UNESCO señala que la calidad, pertinencia e internacionalización se deben entender multidimensionalmente -calidad del personal docente, de los programas académicos, de los estudiantes-; pertinencia la forma en que la universidad responde a las necesidades económicas, sociales y culturales de su entorno; e internacionalización entendida tanto en lo que significa el carácter universal del conocimiento como los actuales procesos de integración económica. Los mecanismos de evaluación y acreditación son entendidos como estrategias para dar respuesta a estos retos.

\section{Conclusión}

Como ya se sostuvo con anterioridad, ser investigador docente en una universidad pública o privada competencias para la reflexión, el análisis, la crítica y el desarrollo de propuestas coherentes con las exigencias del entorno. Esto requiere de empezar a desaprender para 
aprender por tanto, la calidad de la enseñanza trasciende la actuación de los profesores. Su capacidad de intervención individualmente, institucionalmente y socialmente es limitada. La actuación docente implica una constante toma de decisiones.

\section{CUADRO 2. MODELO DE DOCENCIA EN EDUCACIÓN SUPERIOR}

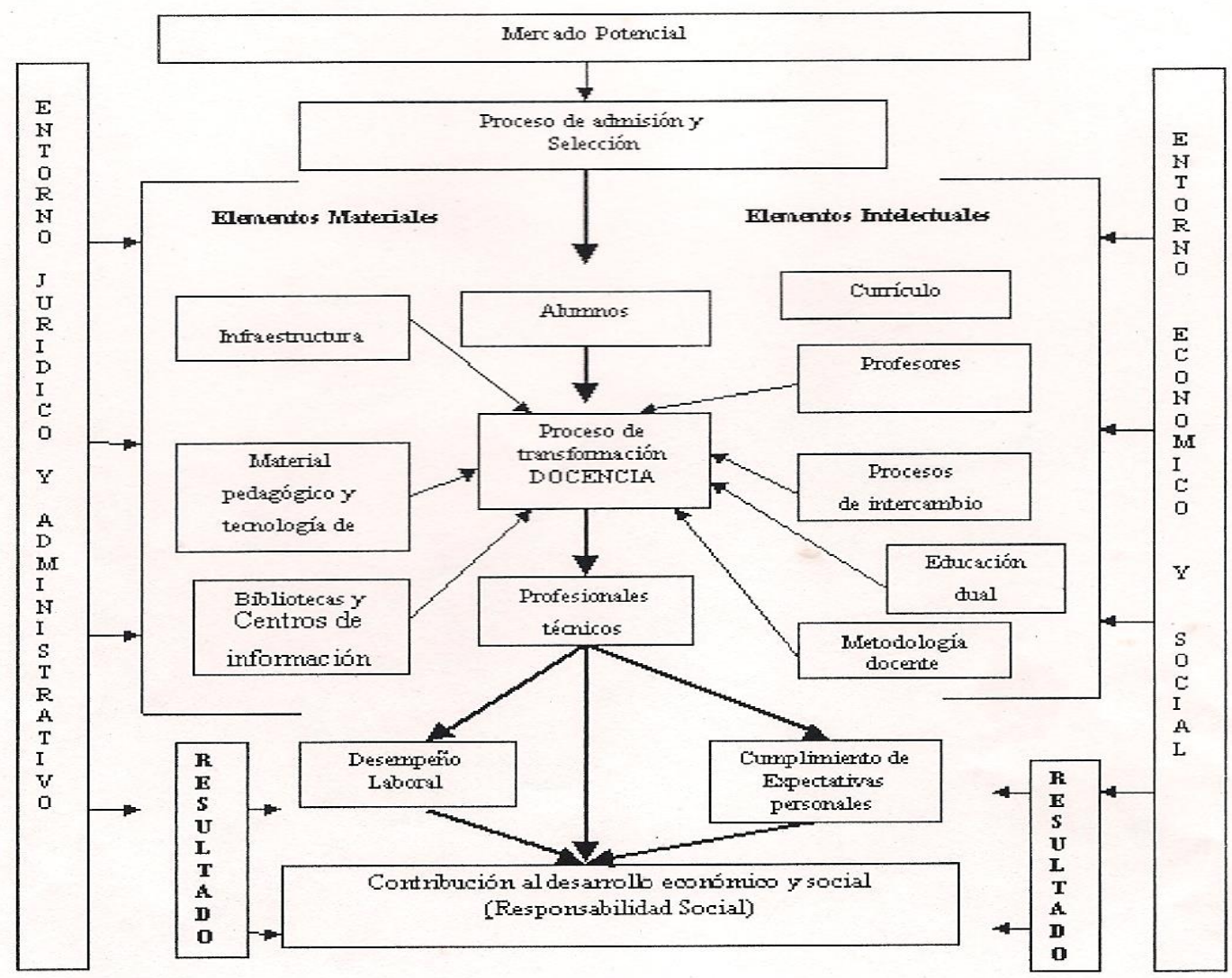

(Méndez y Alarcón: 2000)

La propuesta de análisis de la presente investigación es que la Calidad de la Educación Superior en México requiere de un desarrollo integral eficiente y eficaz que logre una visión holística mediante los siguientes rubros:

1) Modelo de calidad educativa (prestigio, recursos, resultados, niveles, currículum, trasformación). 


\section{CUADRO 3. MODELOS DE CALIDAD}
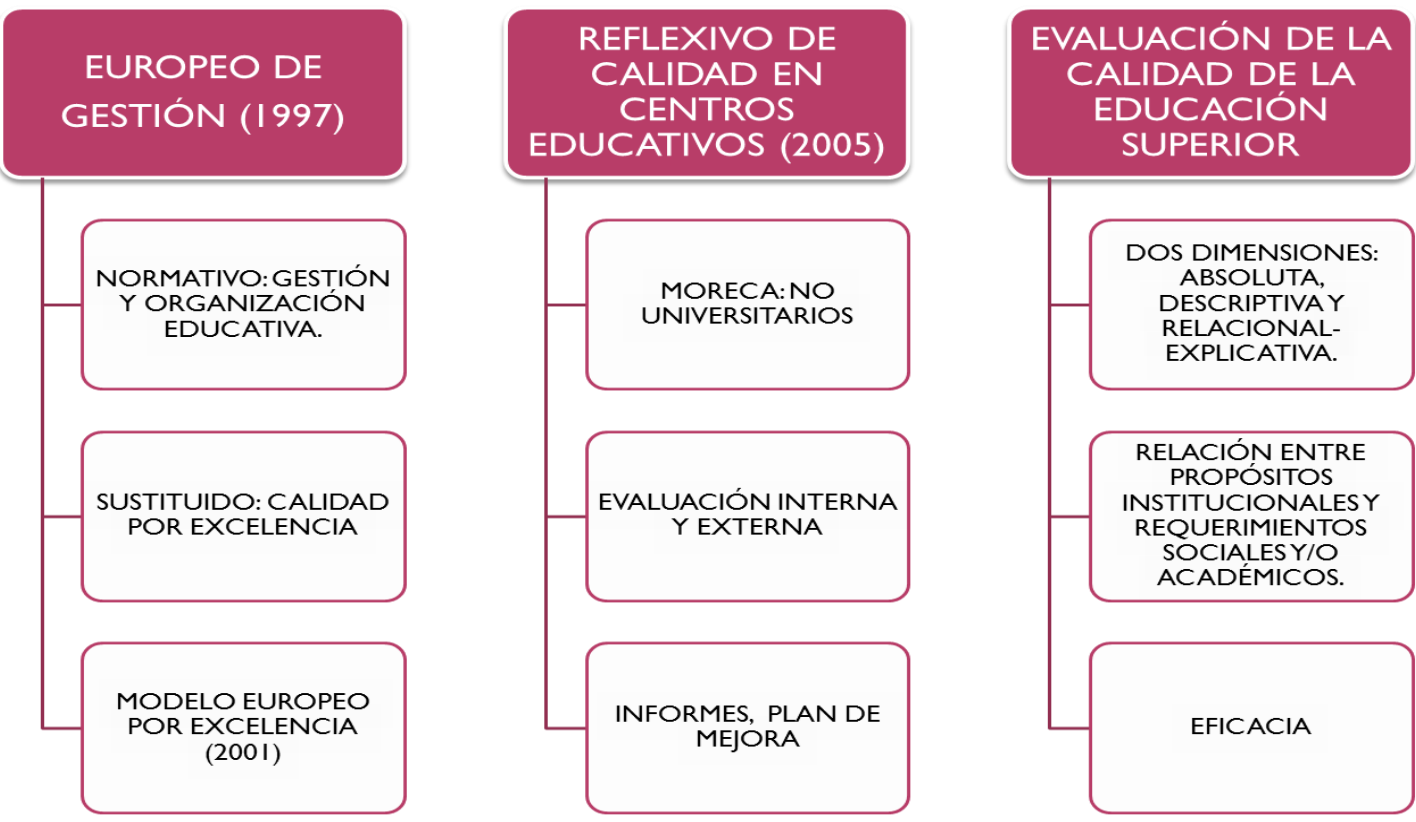

CUADRO 4. MODELOS DE CALIDAD ${ }^{\text {Elaboración propia con información de Cong: 2008) }}$
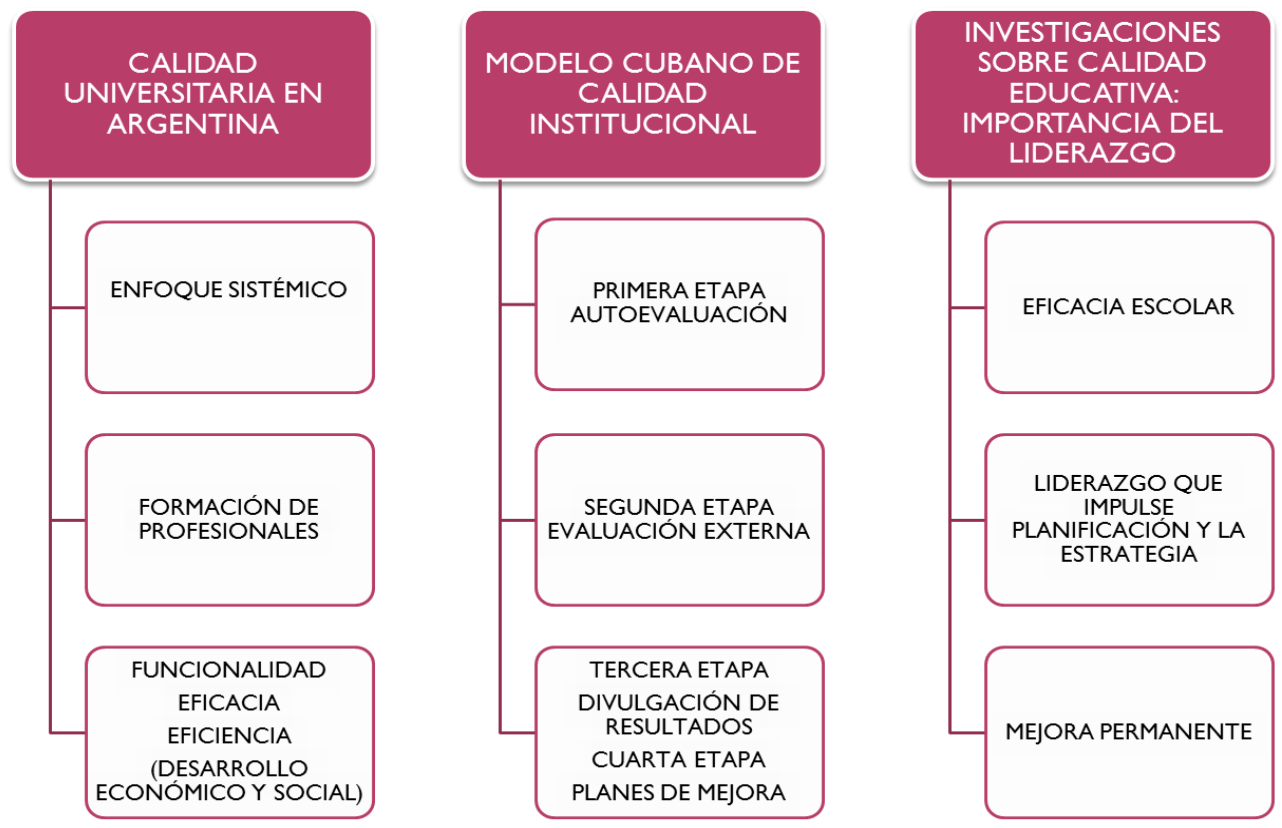

(Elaboración propia con información de Cong: 2008)

2) Modelo educativo (propio de cada institución).

3) Modelo de evaluación de calidad (americano, europeo, británico). 
- 4) Competitividad, competencias y dimensiones de integración disciplinar (por ejemplo el Índice de Competitividad Global (GCl) (World Economic Forum).

\section{CUADRO 5. CALIDAD DE LA EDUCACIÓN SUPERIOR EN MÉXICO}

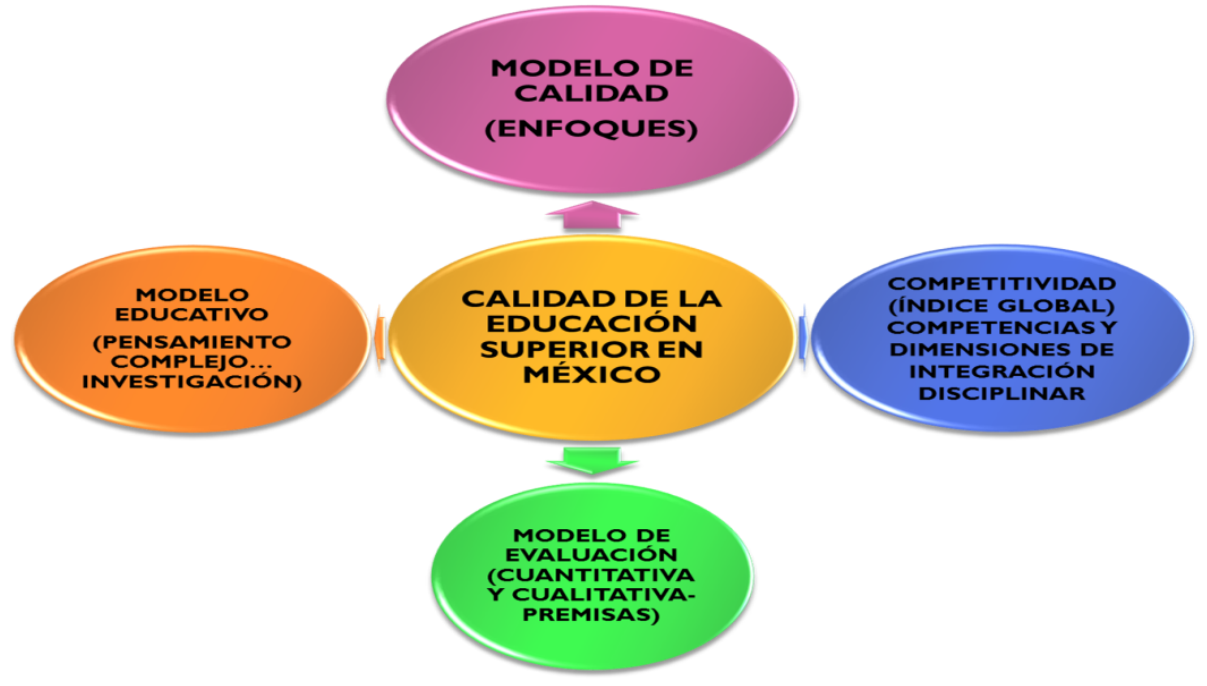

(Mendieta, 2012).

\section{Bibliografía}

ANUIES, (1989). Declaraciones y Aportaciones de la ANUIES para la Modernización de la Educación Superior, Revista de la Educación Superior, 70.

Bazdresch Parada, M. (1996). Evaluación y Calidad en Educación Superior. Aproximación a una Vinculación Necesaria, Reforma y Utopía. Universidad de Guadalajara, México.

Chase, R. \& Aquilano, N. (1995), Dirección y administración de la producción y de las operaciones. Barcelona: Editorial IRWIN.

Díaz Barriga, F. \& Hernández, G. (2002). Estrategias docentes para un aprendizaje significativo. Una interpretación constructivista. México: McGraw Hill.

Diestro Fernández, A. (2003). Calidad y principios educativos. Necesidad de una pedagogía para todos. Revista Foro de Educación, 1. 
Martínez Rizo, F., (1992), La Calidad de las Instituciones de Educación Superior. Su Evaluación y su Promoción, Cuaderno de Planeación Universitaria, UNAM, México.

Martínez Rizo F., (1996), Estrategias de Búsqueda de Calidad en las Instituciones de Educación Superior, Reforma y Utopía, Universidad de Guadalajara, México.

Morin Edgar (1990). Introducción al pensamiento complejo. Madrid: Gedisa.

Pallán Figueroa, C. \& Van der Donck, P. (1995). Evaluación de la Calidad y Gestión del Cambio. México: ANUIES.

Woolfolk A. (1999). Psicología educativa, México: Prentice Hall. 